\title{
Impacto de la pandemia COVID 19 en las condiciones ambientales
}

\section{Impact of the COVID 19 pandemic on environmental conditions}

\author{
Manuel Emilio Milla Pino ${ }^{1}$ (D), María Marleni Torres Cruz $^{2^{*}}$ (D) Cristian Jesús Rojas Milla $^{3}$ (D)
}

\section{RESUMEN}

La presente nota científica tiene como propósito discutir el impacto de la pandemia COVID 19 en las condiciones ambientales, expresada en términos de cómo las medidas estratégicas implementadas por el estado, específicamente el aislamiento social obligatorio, ha incidido en aspectos, tales como, agua de los ríos, condiciones atmosféricas, especies vegetales, especies animales y generación de residuos. Este análisis permite concluir que las medidas de confinamiento han favorecido a la disminución de los niveles de contaminación, evidenciado a través de la mayor presencia de peces en los ríos, claridad de la atmósfera, verdor y regeneración de especies vegetales, y aumento del volumen de residuos no reutilizables.

Palabras clave: COVID 19, condiciones ambientales, generación de residuos.

\begin{abstract}
The purpose of this scientific note is to discuss the impact of the COVID 19 pandemic on environmental conditions, expressed in terms of how the strategic measures implemented by the state, specifically mandatory social isolation, have affected aspects, such as water from rivers, atmospheric conditions, plant species, animal species and waste generation. This analysis allows us to conclude that the confinement measures have favored the decrease in pollution levels, evidenced by the greater presence of fish in rivers, clarity of the atmosphere, greenery and regeneration of plant species, and an increase in the volume of non-reusable waste.
\end{abstract}

Keywords: COVID 19, environmental conditions, waste generation.

\footnotetext{
${ }^{1}$ Universidad Nacional de Jaén, Facultad de Ingeniería Civil, Jaén, Perú.

${ }^{2}$ Universidad Nacional de Jaén, Facultad de Ingeniería Forestal y Ambiental, Jaén, Perú.

${ }^{3}$ Facultad de Ciencias Básicas, Universidad del Atlántico, Barranquilla, Colombia.

*Autor de correspondencia. E-mail: marle.torres@unj.edu.pe
} 


\section{INTRODUCCIÓN}

Aun cuando las medidas de aislamiento social obligatorio implementadas por el estado tienen como propósito fundamental detener la propagación de la pandemia COVID 19, las mismas generan un impacto en las condiciones ambientales, cuyo abordaje constituye el eje central de este trabajo, el cual está orientado hacia la discusión del referido impacto en cinco ejes prioritarios: agua de los ríos, condiciones atmosféricas, especies vegetales, especies animales y generación de residuos.

\section{DESARROLLO}

\section{Agua de los ríos}

En cuanto a las corrientes de agua de los ríos, podemos señalar que el confinamiento social obligatorio ha generado restricciones que impiden la normal dinámica de la población, lo que se traduce en una menor cantidad de personas interactuando en los cauces de los ríos, aspecto que disminuye los niveles de contaminación aumentando así la cantidad de oxígeno que puede ser aprovechada por los seres vivos acuáticos para lograr su subsistencia y desarrollo, hecho que se evidencia cuando observamos un incremento en la cantidad de peces, lo cual es una señal del beneficio que representa el oxígeno.

Existen hallazgos experimentales que sustentan lo antes expuesto, tales como los resultados emanados de la Autoridad Nacional de Agua (ANA), sobre estudios realizados en las regiones hidrográficas (R.H) del Pacífico, Amazonas y Titicaca, así como un cuerpo marino costero, que revelan, que de 32 puntos muestreados, 26 presentaron variaciones positivas en cuanto a los niveles de salinidad y oxigenación y seis presentaron acercamientos al rango de $\mathrm{pH}$ permitido en los ECA para Agua (MIDAGRI, 2021).

En este sentido, los cuerpos de agua que registran niveles de salinidad inferiores al límite máximo permisible establecido en la normativa ambiental, son los ríos Olmos, Chillón, Lurín, Ocoña, Torata, Moquegua y Locumba, así como los ríos Amazonas y Acre. Un caso particular lo representa el río Locumba, el cual antes de decretarse el estado de emergencia tenía valo- res de salinidad que incumplían el ECA para agua y en la actualidad ha mejorado sustancialmente logrando valores acordes con la normativa ambiental vigente.

Es oportuno señalar, que los ríos Lurín y Chillón, Chumbao, Huatanay, Huacarpay, Tambopata y Huancané, tuvieron un ascenso en sus niveles de oxigenación, lo que les permite cumplir con los ECA para agua, lo que constituye un aspecto positivo, por lo beneficioso de este hecho para el desarrollo de los organismos acuáticos, resaltando que antes de la pandemia estos ríos no presentaban valores de oxigenación que cumplieran con la normativa ambiental.

En este orden de ideas, la APN (2021) informó que el río Rímac mejoró en su coloración y su autodepuración. Sin embargo, desde que se levantó la cuarentena ha vuelto a ser ensuciada.

\section{Condiciones atmosféricas}

Las restricciones impuestas a raíz de la pandemia COVID 19, tal como el aislamiento social obligatorio, han disminuido sustancialmente el uso del parque automotor, así como también la operatividad de las plantas industriales encargadas de transformar la materia prima en productos y subproductos, aspectos que se traducen en una menor cantidad de gases contaminantes depositados en la atmósfera, lo cual se evidencia ya que en la actualidad se observa un ambiente más claro y un aire más puro que facilita la respiración de los seres vivos. Al respecto, el SENAMHI (2020) informa que debido al estado de emergencia a causa de la pandemia producida por el coronavirus del COVID - 19, se ha reducido la concentración de contaminantes particulados en el aire de Lima Metropolitana.

Con mayor precisión, se puede afirmar que las concentraciones del $\mathrm{PM}_{2.5}$ han ido descendiendo progresivamente, motivado a por las medidas que restringen la movilidad implementadas por el Estado. Antes de la pandemia se observaban valores en las estaciones San Juan de Lurigancho, Campo de Marte y Villa María del Triunfo, que al ser comparados con las concentraciones actuales reflejan una reducción de la contaminación de $72 \%, 29 \%$ y $43 \%$, respectivamente, lo que sin duda alguna está significativamente asociado con la 
prohibición del uso de vehículos establecida en el marco de la pandemia.

Así mismo, se puede señalar que a medida que se han ido flexibilizando las acciones decretadas para el control de la pandemia, se observa un incremento en los valores de los índices que estiman la reducción de la contaminación, lo cual está relacionado con el aumento progresivo del uso de las unidades que integran el parque automotor y la reactivación económica que implica que una mayor cantidad de empresas y plantan industriales entren en funcionamiento.

\section{Especies vegetales}

En lo referente al desarrollo de las especies vegetales se observa mayor verdor en las plantas, lo cual puede obedecer a que hay menor cantidad de desperdicios o desechos en el suelo, lo que indica que el agua suministrada a las plantas por vía natural o artificial tiene mayor posibilidad de ser aprovechada o adsorbida a través de las raíces de las mismas, y esto se traduce en un mejor desarrollo, evidenciado por medio de la frondosidad y la biomasa.

También se observa que en zonas donde antes de la pandemia había limitaciones para el desarrollo de ciertas especies, actualmente esas especies se han ido regenerando, lo cual puede ser debido a la poca acción destructiva del hombre, motivado a que el confinamiento obligatorio restringe su contacto con la naturaleza.

Este impacto positivo en el medio ambiente es gracias a la ausencia del ser humano y a la reducción de la mayoría de los procesos productivos y extractivos que se dan a diario mundialmente. Sabemos que después de este periodo de crisis sanitaria mundial, el estilo de vida no será el mismo (Gómez, 2021).

Las especies vegetales se han visto beneficiadas por el aislamiento social obligatorio, ya que esto ha restringido el dinamismo social motivado por la poca movilidad autorizada por los órganos del estado, lo que en consecuencia ha permitido el fortalecimiento, desarrollo y regeneración de las especies vegetales, aprovechando la existencia de un menor contacto con los sujetos que integra la población. De ahí que esto se evidencia a través del verdor de las plantas, así como también el desarrollo de especies vegetales que parecían estar en extinción.

En este contexto encontramos importantes resultados en el estudio de especies vegetales, tal es el caso, de investigadores que eligieron tres pares de poblaciones simpáticas de las dos especies de Nepal, Camboya y Laos para realizar en ellas 238 combinaciones de cruces artificiales, incluidas las combinaciones entre especies, entre poblaciones dentro de especies y entre individuos dentro de poblaciones. Sobre esta base, estimaron el conjunto de semillas de polinizaciones cruzadas, la viabilidad F1 y la fertilidad para evaluar el aislamiento reproductivo post-cigótico. Además, los investigadores recolectaron los datos del tiempo de floración de estas poblaciones censurados en su experimento común anterior para evaluar el aislamiento reproductivo precigótico (Mundo Agropecuario, 2021).

\section{Especies animales}

Las restricciones impuestas por el estado, en cuanto a la prohibición de salida en ciertas horas del día a traído como consecuencia un menor contacto de la población con las especies animales en aquellos espacios aptos para el desarrollo de estos seres vivos. Por lo tanto, a medida que ha transcurrido la pandemia, han comenzado aparecer especies animales que antes de la pandemia no era común visualizar y esto puede estar relacionado con el hecho de que los menores niveles de contaminación generan espacios acordes con los requerimientos de calidad ambiental que ciertas especies animales necesitan para su desarrollo.

En distintas partes del mundo se ha podido observar la aparición de fauna silvestre en lugares que antes era imposible encontrarlos: leones durmiendo en las carreteras de Sudáfrica, jabalíes en calles de Madrid, sajinos en calles de Colombia. En nuestro país se han observado distintas especies de aves en las playas de la Costa Verde e incluso la aparición de delfines (Gómez, 2021). Ante el confinamiento la fauna está ganando terreno. En los espacios naturales ya no hay escaladores ni senderistas, no hay vuelos en parapente, ni en globo o en helicóptero y apenas pasan aviones ni vehículos a motor. Mucho menos cazadores. Los expertos de algu- 
nas organizaciones de protección de la fauna consideran que lo que está sucediendo tiene un efecto sanador para muchas especies en peligro dentro de la geografía mundial, como el quebrantahuesos, las águilas, el oso pardo, el gato montés o el lobo ibérico. "Estoy seguro de que se incrementarán los avistamientos de especies que antes eran casi invisibles como el licaón, o el rinoceronte negro en África, y algunos tipos de ballenas en Galápagos”, afirma Jordi Serrallonga, arqueólogo, naturalista y profesor de la Universitat Oberta de Catalunya. "El confinamiento ha desembocado en la rebelión o libertad animal” (Martínez-Mansilla, 2021).

Un reflejo de los antes expuesto se puede apreciar en las redes sociales, en las cuales se muestran ciertas especies animales circulando por espacios donde antes de la pandemia era imposible observarlos, tal es el caso, de delfines en las costas del Levante, un oso pardo paseando por las calles de Ventanueva en Asturias y un jabalí por las de Barcelona, pavos reales en Madrid, cabras montesas al galope en Chinchillas (Albacete) y hasta una foca en la ribera de San Sebastián.

\section{Generación de residuos}

Es conveniente reconocer que el asilamiento obligatorio debido a la pandemia, ha generado impactos positivos en el ecosistema, pero observamos algunos residuos sólidos que antes no se generaban, tal es el caso de las mascarillas, guantes y descartables. Estos residuos, al no contar con un manejo adecuado y sumado a las altas cantidades diarias, podrían conducir a niveles significativos de contaminación, ya que no logran degradarse fácilmente e incorporarse al ambiente.

Al respecto, SPDA (2021), informó que, tras el inicio de la cuarentena focalizada, se registró un mayor número de residuos domésticos y de construcción en las fuentes naturales de agua como ríos, canales de regadío y drenes. Asimismo, resaltó el aumento de la presencia de mascarillas y guantes en los lugares mencionados.

En líneas generales se viene observando un aumento progresivo en la producción y uso de material plástico, resaltando aquellos que no son reutilizables; aspecto que conduce a pensar que a medida que continúe el confinamiento en esa misma medida se incrementará el volumen de plástico consumido.

Gran parte de los equipos de protección que utilizan los trabajadores de la salud - como guantes, mascarillas y batas - son de un solo uso. Sin embargo, son residuos plásticos considerados peligrosos porque se disponen en hospitales. Hoy, la historia es diferente porque encontramos estos residuos en casa y se han convertido en residuos domésticos (PUCP, 2021). Es indudable que la pandemia ha incrementado el consumo de todo tipo de plástico, muy especialmente, los desechables, como son las bolsas, botellas de agua y recipientes para comida delivery. Por un tema de salud pública con énfasis en el cuidado, las personas han aumentado el uso de polietileno, pero la disposición de los desechos no es la adecuada, ya que los residuos o desperdicios son colocados en cualquier lugar distinto a los contenedores dispuestos para tal fin.

Además del uso de mascarillas y guantes, también se observa el uso de geles alcohólicos y toallitas desinfectantes, lo que genera un incremento en los residuos, aspecto que, aunado al manejo inadecuado de estos, los convierte en un foco de contaminación ambiental.

La pandemia de COVID-19 también ha aumentado el consumo de otros plásticos desechables como bolsas, botellas de agua, recipientes para enviar comida a domicilio o embalajes del comercio por internet. Un informe de Ecoembes señala que desde el inicio del estado de alarma se ha incrementado un $15 \%$ la recogida de material en los contenedores amarillos. Este aumento se debe a un mayor consumo dentro de los hogares como consecuencia del confinamiento de la población (Eljarrat, 2021).

\section{CONCLUSIÓN}

Las medidas de confinamiento han favorecido a la disminución de los niveles de contaminación, evidenciado a través de la mayor presencia de peces en los ríos, claridad de la atmósfera, verdor y regeneración de especies vegetales, así como el aumento del volumen de residuos no reutilizables, motivado a las restricciones establecidas para la movilidad social. 


\section{CONTRIBUCIÓN DE LOS AUTORES}

Manuel Emilio Milla Pino, fue el encargado de la redacción del documento y adecuación a las normas de la revista. María Marleni Torres Cruz fue la responsable de la recopilación de información referida al tema en estudio. Cristian Jesús Rojas Milla realizó la sistematización de la información.

\section{CONFLICTO DE INTERESES}

Los autores declaran no tener conflicto de intereses.

\section{REFERENCIAS BIBLIOGRÁFICAS}

APN (Agencia Peruana de Noticias). 2021. Agencia Peruana de Noticias. https://andina.pe/agencia/noticia-alertanaumento-residuos-como-mascarillas-yguantes-rios-y-canales-regadio-806426.aspx (Consultada el 11 de marzo del 2021).

Eljarrat, E . 2021. Daños colaterales de la COVID-19: el resurgir del plástico. https://theconversation.com/danoscolaterales-de-la-covid-19-el-resurgir-delplastico-137803 (Consultada el 11 de marzo del 2021).

Gómez, M. 2021. Columna de Opinión: Impactos positivos de la cuarentena en el medio $a m b i e n t e \quad y \quad p l a n e t a$. https://noticias.upc.edu.pe/2020/04/21/colu mna-opinion-impactos-positivos-de-lacuarentena-1-medio-ambiente-planeta/ (Consultada el 11 de marzo del 2021).

Martínez-Mansilla, J. 2021.¿Qué consecuencias tiene el confinamiento para la naturaleza? https://www.traveler.es/naturaleza/articulos/ el-medio-ambiente-y-el-coronavirus-queconsecuencias-tiene-el-confinamiento-parala-naturaleza/17843 ( Consultada el 11 de marzo del 2021).

MIDAGRI (Ministerio de Desarrollo Agrario y Riego). 2021. Autoridad Nacional del Agua. https://www.ana.gob.pe/noticia/aumentanresiduos-como-mascarillas-y-guantes-en- rios-y-canales-de-regadio (Consultada el 11 de marzo del 2021).

Mundo Agropecuario . 2021. Mundo Agropecuario se mbrando el desarrollo. https://mundoagropecuario.com/ladivergencia-en-el-tiempo-de-floracioncontribuye-al-aislamiento-reproductivoentre-las-especies-de-arroz-silvestre (Consultada el 11 de marzo del 2021).

PUCP (Pontificia Universidad Católica del Perú). 2021 . Clima de cambios. https://www.pucp.edu.pe/climadecambios/n oticias/covid-19-el-resurgir-del-plastico (Consultada el 11 de marzo del 2021).

SENAMHI (Servicio Nacional de Meteorología e Hidrología del Perú). 2020. Mejora en la calidad de aire de Lima Metropolitana. https://www.gob.pe/institucion/senamhi/noti cias/127454-mejora-en-la-calidad-de-airede-lima-metropolitana (Consultada el 11 de marzo del 2021)

SPDA (Sociedad Peruana de Derecho Ambiental). 2021. ANA: residuos como mascarillas y guantes aumentaron en ríos y canales de $\begin{array}{lllllll}r & e & g & a & d & i & o\end{array}$. https://www.actualidadambiental.pe/anaresiduos-como-mascarillas-y-guantesaumentaron-en-rios-y-canales-de-regadio (Consultada el 11 de marzo del 2021) 